\title{
Structure Optimization of Safety Investment of Petrochemical Port Enterprises
}

\author{
Zhiqiang Hou ${ }^{1,2}$ and Peng Zhao \\ ${ }^{1}$ School of Traffic and Transportation, Beijing Jiaotong University, Beijing 100044, China \\ ${ }^{2}$ China Waterborne Transport Research Institute, Beijing 100088, China \\ Correspondence should be addressed to Zhiqiang Hou; zhiqianghou@163.com
}

Received 20 March 2017; Accepted 10 May 2017; Published 30 May 2017

Academic Editor: Chaudry M. Khalique

Copyright (C) 2017 Zhiqiang Hou and Peng Zhao. This is an open access article distributed under the Creative Commons Attribution License, which permits unrestricted use, distribution, and reproduction in any medium, provided the original work is properly cited.

\begin{abstract}
Safety investment is an essential guarantee to identify and manage potential security problems in petrochemical port enterprises. The reasonability of safety investment structure determines overall security risks in an enterprise. Based on the definition of risks, combining Cobb-Douglas production function with FTA probability model, and taking Gompertz curve model as the constraint condition, structure optimization model of safety investment is built in order to minimize risks and work out the safety investment structure of petrochemical port enterprises. According to the case study, the calculations indicate that safety investment in corporate management presents a larger growth rate than past years and that unsafe act of human being is the main factor accounting for the greatest probability of occurrence, which is consistent with previous accident investigation results as well as enterprise reality. This testifies that the model is effective and that the results can guide the allocation of safety investment of petrochemical port enterprises scientifically.
\end{abstract}

\section{Introduction}

Recently, explosion accidents happening in Dongjiang Free Trade Port Zone of Tianjin, New Dalian Port, and Gulei Port of Zhangzhou, as well as other petrochemical ports have aroused the attention and concern of both the government and the society. The investigation results show that unreasonable safety investment structure is one of the main reasons for accidents. Safety investment is an essential guarantee to identify and manage potential security problems. How to allocate investment with limited funds in petrochemical port enterprises is one of the key issues for waterway transportation industry.

There are many domestic and foreign literatures on structure optimization of safety investment of manufacturing enterprises. Most of them focused on industries such as coalmining, construction, and iron and steel whereas few on waterway transportation industry are composed currently. Nie et al. built a model analyzing the relationship between safety investment and safety in production based on fuzzy evaluation and concluded that a linear relationship existed between the two. The model can improve the accuracy of comprehensive fuzzy evaluation currently used [1]. Son et al. applied cost-benefit analysis to research on optimum structure of safety investment in construction industry [2]. Lei and Yuanyuan testified the relationship between safety investment and accident cost in coal-mining industry with the assistance of system dynamics method [3]. Hai-zhi et al. built an optimized model aiming at a minimal safety investment and believed that an optimized allocation among various safety investment items could be reached within a given safety investment [4]. Zhong et al. established an optimal control model of corporate safety cost according to nonlinear optimization theory and analyzed the cost of an iron and steel enterprise. The result showed that safety cost and accident loss were both lower than average [5]. You-song et al. built a safety investment model on falling accidents in construction industry and realized the optimized allocation among detailed items of safety investment [6].

Research methods frequently applied in domestic and foreign studies on structure optimization of safety investment 
are mainly fuzzy evaluation, optimization model of minimal safety cost, and optimal model of safety benefits. Fuzzy evaluation relies mainly on surveys conducted by experts and therefore may be subjective and limited by knowledge, experience, logical thinking, and judgement of experts. Optimization model of minimal safety cost and optimal model of safety benefits both focus on the minimal sum of safety investment and accident loss. Safety benefits are considered to be optimal under such circumstance and the amounts of various items of safety investment are calculated accordingly. Actually, the fundamental aim of safety investment is to lower safety risks. The minimal safety cost or optimal safety benefits may not reduce safety risks effectively. Even though the total amount of safety investment in petrochemical port enterprises meets the requirements of relevant documents, an unbalanced investment among diverse items may probably cause a bigger risk beyond the acceptable range due to insufficient investment in certain aspects [7]. Therefore, it is necessary to allocate safety investment funds scientifically to lower the overall risks in petrochemical port enterprises.

Risk is the product of accident probability and accident loss. Cobb-Douglas production function can be represented by the functional relationship between accident loss and items of safety investment. The solution for FTA top event expresses the functional relationship between accident probability and probability of basic events. Gompertz curve can be used to establish the functional relationship between safety investment and accident probability of items. In consideration of the conditions above, an optimized structure model of safety investment aiming at minimal risks is proposed to work out the safety investment structure and accident probability of items. The effectiveness of optimized model proposed is verified by practical examples in this paper.

\section{Structure Optimization Model of Safety Investment of Petrochemical Port Enterprises}

2.1. Model of Accident Loss and Safety Investment. There is a functional relationship between accident loss and safety investment. The inner law between accidental economic loss and safety investment of items can be represented by CobbDouglas production function $[8,9]$ in the following way:

$$
y=F\left(x_{1}, x_{2}, \ldots, x_{i}, \ldots, x_{n}\right)=\alpha x_{1}^{\beta_{1}} x_{2}^{\beta_{2}} \cdots x_{i}^{\beta_{i}} \cdots x_{n}^{\beta_{n}},
$$

where $y$ is accident loss, $x_{i}$ is safety investment of different items with a total number of $i$, and $\beta_{i}$ is production elastic coefficient of different items.

It can be concluded according to practical situations and formula (1) that

(1) once accidents happen, there must be accident loss; that is, $y>0$;

(2) generally, accident loss will decrease when the safety investment of a certain item is increased; that is,

$$
\frac{\partial F}{\partial x_{i}}=\alpha x_{1}^{\beta_{1}} x_{2}^{\beta_{2}} \cdots \beta_{i} x_{i}^{\beta_{i}-1} \cdots x_{n}^{\beta_{n}}=\beta_{i} \frac{y}{x_{i}}<0,
$$

therefore, $\beta_{i}<0$;
(3) a reasonable safety investment can lower the probability of risks to an acceptable degree, but risks cannot be eliminated radically by enterprises; that is, $\lim _{k_{i} \rightarrow 0} x_{i}=+\infty$, where $k_{i}$ is accident probability.

2.2. Model of Safety Investment and Accident Probability. According to the analysis above, when accident probability is close to zero, safety investment goes to infinity with a negative correlation between the two. Meanwhile, with safety investment increasing, the decreasing rate of accident probability slows down. This is in accordance with the features of production function curve and Gompertz curve $[10,11]$. In Gompertz curve model, if $k_{i}^{\prime}$ represents accident probabilities of different kinds and $x_{i}^{\prime}$ represents safety investment of different items, then

$$
k_{i}^{\prime}=c_{i} a_{i}^{b_{i}^{x_{i}^{\prime}}}
$$

where $i=1,2, \ldots, n ; c_{i}, a_{i}$, and $b_{i}$ are parameters with $0<a_{i}<$ 1 and $b_{i}>1$. $\left.\ln a_{i}\right)$.

Transform formula (3) and get $x_{i}^{\prime}=\left(1 / \ln b_{i}\right) \ln \left(\ln \left(k_{i}^{\prime} / c_{i}\right) /\right.$

Suppose the total amount of safety investment is $Q$; then

$$
\sum_{i=1}^{n} x_{i}^{\prime}=\sum_{i=1}^{n} \frac{1}{\ln b_{i}} \ln \frac{\ln \left(k_{i}^{\prime} / c_{i}\right)}{\ln a_{i}}=Q,
$$

where parameters $c_{i}, a_{i}$, and $b_{i}$ are defined by existing data.

2.3. Accident Probability Model. Logical reasoning of any accident in petrochemical port enterprises is conducted based on FTA. Direct cause and basic events can be drawn to reveal potential reasons for accidents. According to FTA, accident probability is

$$
p\left(x_{1}, x_{2}, \ldots, x_{i}\right)=\prod_{j=1}^{P}\left[1-\prod_{X_{i} \in p_{j}}\left(1-k_{i}\right)\right],
$$

where $k_{i}$ is the probability of basic events $x_{i}$ in FTA, $P$ is the number of path sets in FTA, and $p_{j}$ is the collection of basic events of different path sets [12].

2.4. Risk Model. In Occupation Health Safety Management System, risk is defined as "the combination of possibility and consequence of a certain dangerous situation" [13]. Safety accident risk in petrochemical port enterprises is the product of accident probability and accident consequence [14]. In this paper, accident loss is taken as accident consequence; combining formulas (1) and (5), the following equation can be reached:

$$
\begin{aligned}
R & =p \cdot y \\
& =\prod_{j=1}^{P}\left[1-\prod_{X_{i} \in p_{j}}\left(1-k_{i}\right)\right] \cdot \alpha x_{1}^{\beta_{1}} x_{2}^{\beta_{2}} \cdots x_{i}^{\beta_{i}} \cdots x_{n}^{\beta_{n}},
\end{aligned}
$$

where $R$ is accident risk, $p$ is accident probability, and $y$ is accident loss. 
2.5. Structure Optimization Model of Safety Investment. Structure optimization model of safety investment is established on the basis of objective function with minimal risk, under restraint condition (4), and presented as follows:

$$
\begin{array}{ll}
\min & R \\
& =\min \left\{\prod_{j=1}^{P}\left[1-\prod_{X_{i} \in p_{j}}\left(1-k_{i}\right)\right] \cdot \alpha x_{1}^{\beta_{1}} x_{2}^{\beta_{2}} \cdots x_{i}^{\beta_{i}} \cdots x_{n}^{\beta_{n}}\right\} \\
\text { st. } \quad & \sum_{i=1}^{n} x_{i}^{\prime}=\sum_{i=1}^{n} \frac{1}{\ln b_{i}} \ln \frac{\ln \left(k_{i}^{\prime} / c_{i}\right)}{\ln a_{i}}=Q, \\
& 0<k_{i}<1 \\
& 0<k_{i}^{\prime}<1 \\
& \beta_{i}<0, \quad i=1,2, \ldots, n .
\end{array}
$$

Model (7) is nonlinear planning model with restraint conditions which can be expanded according to practical situation in petrochemical port enterprises. The analysis of variables in model (7) is as follows.

(1) $k_{i}$ are probabilities of basic events in FTA. If $x_{i}$ and $x_{i}^{\prime}$ are both correspondent safety investments, then $x_{i}=x_{i}^{\prime}, k_{i}=$ $k_{i}^{\prime}$. Combine formulas (7) and (3) and the values of $x_{i}$ and $k_{i}$ can be solved. Finally, the total amount of investment in items is calculated through classifying and summing up $x_{i}$. On this condition, genetic algorithm (GA), simulated annealing (SA), and other intelligent optimization algorithms can be applied to calculate the value.

(2) If the values of $x_{i}$ which represents the probabilities of basic events or the value of $p\left(x_{1}, x_{2}, \ldots, x_{i}\right)$ is known, then $\prod_{j=1}^{P}\left[1-\prod_{X_{i} \in p_{j}}\left(1-k_{i}\right)\right]$ becomes constant. The model can be simplified. Meanwhile, $x_{i}$ may not be correspondent to $k_{i}$. Both $x_{i}$ and $x_{i}^{\prime}$ represent safety investments of items and $k_{i}$ are correspondent probabilities. Then it can be concluded that $x_{i}=x_{i}^{\prime}$ and $k_{i} \neq k_{i}^{\prime}$. In this condition, genetic algorithm (GA), simulated annealing (SA), and other intelligent optimization algorithms can be applied to work the value. It is the same with fmincon function in Matlab [15].

\section{Empirical Analysis}

The handling and shaping goods in the petrochemical port enterprise in the north gulf belong to dangerous goods of Class A. The typical potential accident is "fire explosion." The enterprise decides to resolve potential security problems centering on fire explosion accidents. According to related regulations, the total amount of safety investment should be no less than $¥ 3033180$ based on the proportion of operating revenues in 2016.

3.1. Classification of Safety Investment. Many factors may trigger fire explosion in petrochemical dock. According to FTA, taking "fire explosion" as top event, basic events of FTA are classified into four categories: $x_{1}$ is safety investment in relevant personnel; $x_{2}$ is safety investment in equipment and
TABLE 1: Safety investment and accident loss in different years (unit: ten thousand).

\begin{tabular}{lcccccc}
\hline Number & Year & $x_{1}$ & $x_{2}$ & $x_{3}$ & $x_{4}$ & $Y$ \\
\hline 1 & 2009 & 69.358 & 151.324 & 41.691 & 0.931 & 8.498 \\
2 & 2010 & 68.731 & 157.690 & 40.320 & 0.939 & 8.517 \\
3 & 2011 & 70.491 & 152.161 & 40.101 & 0.823 & 8.611 \\
4 & 2012 & 72.631 & 167.976 & 42.110 & 0.901 & 8.462 \\
5 & 2013 & 68.125 & 150.437 & 41.098 & 0.741 & 8.709 \\
6 & 2014 & 85.195 & 172.327 & 51.562 & 0.902 & 8.155 \\
7 & 2015 & 64.178 & 156.142 & 37.163 & 0.792 & 8.781 \\
\hline
\end{tabular}

TABLE 2: Coefficients of Gompertz curve.

\begin{tabular}{lccc}
\hline Incident code & $c_{i}$ & $a_{i}$ & $b_{i}$ \\
\hline$x_{1}$ & 0.070 & 0.950 & 1.070 \\
$x_{2}$ & 0.003 & 0.398 & 1.009 \\
$x_{3}$ & 0.080 & 0.110 & 1.011 \\
$x_{4}$ & 0.040 & 0.150 & 5.010 \\
\hline
\end{tabular}

facilities; $x_{3}$ is safety investment in management; $x_{4}$ is safety investment in environmental enhancement.

3.2. Function Fitting and Model Parameters Determination. The allocation of safety investment in managing potential security problems as well as accident loss is stated in Table 1.

The natural logarithms of the two sides of formula (1) are presented as follows: transforming multivariate nonlinear equation to multivariate linear equation, that is,

$$
\begin{aligned}
\ln y= & \ln \alpha+\beta_{1} \ln x_{1}+\beta_{2} \ln x_{2}+\cdots+\beta_{i} \ln x_{i}+\cdots \\
& +\beta_{n} \ln x_{n} .
\end{aligned}
$$

Multiple linear regression analysis of Table 1 is conducted transforming it into multivariate nonlinear function. The model of accident loss and safety investment is presented as follows:

$$
\begin{aligned}
y & =F\left(x_{1}, x_{2}, x_{3}, x_{4}\right) \\
& =20.4644 x_{1}^{-0.1027} x_{2}^{-0.0191} x_{3}^{-0.0949} x_{4}^{-0.0924} .
\end{aligned}
$$

3.3. Gompertz Curve Fitting. Gompertz curve fitting is conducted and the values of $c_{i}, a_{i}$, and $b_{i}$ are calculated based on Delphi Method [16, 17], combining relevant documents on corporate security accident, as shown in Table 2.

3.4. Model Solving. Catastrophic fire explosion happened in Tianjin Port on August 12, 2015. In this case, according to requirements of superior authorities, the petrochemical enterprises aim to maintain existing safety facilities and bring in another series of safety equipment, with the investment budget no less than $¥ 1708500$ in 2016. Meanwhile, 
TABLE 3: Optimal safety investments in 2016 and 2015 (unit: ten thousand).

\begin{tabular}{lccccc}
\hline Number & Items of safety investment & Optimum value & Investment in 2015 & Difference & Difference rate\% \\
\hline 1 & Safety investment in personnel & 65.774 & 64.178 & 1.596 & 2.49 \\
2 & Safety investment in facilities & 170.85 & 156.142 & 14.708 & 9.42 \\
3 & Safety investment in management & 65.755 & 37.163 & 28.592 & 76.94 \\
4 & Safety investment in environmental enhancement & 0.939 & 0.792 & 0.147 & 18.56 \\
\hline
\end{tabular}

the investment in environmental enhancement is no less than that of the maximum in Table 1 . Based on the restraint conditions above, structure optimization model of safety investment can be established as follows:

$$
\begin{array}{ll}
\min & R=\min \left\{\prod_{j=1}^{P}\left[1-\prod_{X_{i} \in p_{j}}\left(1-k_{i}\right)\right] \cdot 20.4644 x_{1}^{-0.1027} x_{2}^{-0.0191} x_{3}^{-0.0949} x_{4}^{-0.0924}\right\} \\
\text { st. } \quad & \sum_{i=1}^{4} x_{i}=\sum_{i=1}^{4} \frac{1}{\ln b_{i}} \ln \frac{\ln \left(k_{i}^{\prime} / c_{i}\right)}{\ln a_{i}}=303.318, \\
& 170.850 \leq x_{2}<303.318, \\
& 0 \leq x_{4} \leq 0.939 .
\end{array}
$$

Since the probability of basic events is given in [18], the values are calculated with the assistance of function fmincon in Matlab. The results of optimal safety investment in 2016 in comparison with those of 2015 are presented in Table 3.

After calculating, the proportions of investment in different items are $x_{1}: x_{2}: x_{3}: x_{4}=0.2168: 0.5633: 0.2168: 0.0031$; the accident probabilities follow the order of $k_{4}<k_{2}<k_{3}<$ $k_{1}$.

3.5. Results Analysis. The difference values in Table 3 are all positive indicating an increase in all items of safety investment in 2016. This is also because the overall safety investment grows by $17.44 \%$ in 2016 compared with that in 2015 . Therefore, it can be concluded that safety investments in personnel $x_{1}$, in equipment and facilities $x_{2}$, and in environment enhancement $x_{4}$ all show a small growth. The largest growth happened to safety investment in management $x_{3}$, with a $76.94 \%$ growth rate compared with that in 2015 . Accordingly, in 2016, the petrochemical enterprises should have expanded safety investment in management including legal management of safety in production, administrative management, supervision and inspection, process technology management, and equipment and facilities management. The lack of safety management is the main cause for accident after analyzing previous fire explosion accidents in petrochemical ports. Therefore, it is imperative to increase safety investment in management to ensure safety in production and operation. The detailed allocation of investment in different items can also be calculated based on the method stated in this paper. In terms of accident probability, human is the main factor followed by management, equipment and facilities, and finally environment, which indicates that a larger accident probability does not necessarily mean a greater risk. Human factor refers to unsafe acts of human being, such as misoperation, misdirection, and operation against regulations. The root cause is actually the problems with safety training, safety education, and safety culture. After visiting more than fifty petrochemical enterprises throughout the country, conclusion can be drawn that most accidents are triggered by unsafe behaviors of human being. The factor of equipment and facilities refers to the unsafe conditions of objects, such as unreasonable design, defects in equipment and facilities, and operation under obvious malfunction. Currently there are various kinds of relevant regulations on safety equipment, special equipment examination, and lightning and thunder prevention test. As long as enterprises follow these regulations and examine and maintain equipment and facilities, the accident probability is relatively low. Environmental factor refers to insufficient lighting, narrow and small working space, and high dust concentration in confined space. Problems above seldom exist in petrochemical port enterprises and the probability of occurrence is low.

\section{Conclusion}

Structure optimization model of safety investment is built aiming at risk minimization, combining Cobb-Douglas production function model, FTA probability model, and Gompertz curve model organically, with the fundamental aim to lower the risk of accidents based on definition of accident risks. In empirical analysis, the calculated results of safety investment structure of a certain enterprise in 2016 show that safety investment in management presents a rapid increase and that unsafe act of human being accounts for the highest 
probability of accident occurrence. Therefore, enterprises should expand safety investment in management and improve personnel training and education to lower the risk and probability of fire explosion accidents and promote use effect of safety investment. However, safety in production is dynamic in petrochemical enterprises. Although the amount of safety investments in different items is made clear, an equal amount of investment may have different effect. Hence, how to make use of safety investment is still an issue needed to be discussed. In addition, the time span of statistics data and the database of different types of accidents may also have impacts on the fitting of model of accident loss and safety investment and on the parameter values of Gompertz curve. Therefore, more data need to be collected further to enhance the effectiveness of the model.

\section{Conflicts of Interest}

The authors declare that they have no conflicts of interest.

\section{References}

[1] B.-S. Nie, P.-F. Zhao, J.-H. Guo et al., "Fuzzy comprehensive evaluation of coal mine safety investment structure based on the M(1,2,3) model," Energy Procedia, vol. 16, pp. 592-597, 2012.

[2] K. S. Son, R. E. Melchers, and W. M. Kal, "Analysis of safety control effectiveness," Reliability Engineering and System Safety, vol. 68, no. 3, pp. 187-194, 2000.

[3] T. Lei and D. Yuanyuan, "Simulation study of coal mine safety investment based on system dynamics," International Journal of Mining Science and Technology, vol. 24, no. 2, pp. 201-205, 2014.

[4] R. Hai-zhi, C. Yu-qin, and C. Lian-jun, "Study on optimizing both size and structure of safety investment in coal enterprises," China Safety Science Journal, vol. 24, no. 8, pp. 3-8, 2014.

[5] Z. Zhong, W. Yu-jie, H. Yu-tao et al., "Optimization control model of enterprise safety cost base on the non-linear programming," Journal of Wuhan University of Technology, vol. 32, no. 11, pp. 141-144, 2009.

[6] W. You-song, Z. Yu-fan, W. Zi-han et al., "Based on the FTA method of the safety investment optimization allocation on prevention for falling accident from high place of construction," Journal of Engineering Management, vol. 28, no. 1, pp. 87-92, 2014.

[7] L. Yun, F. Yun-xiao, and M. Xiao-chun, Risk Analysis and Safety Evaluation, Chemical Industry Press, Beijing, 2014.

[8] A. Nikkhah, B. Emadi, and H. Soltanali, "Integration of life cycle assessment and cobb-douglas modeling for the environmental assessment of kiwifruit in Iran," Journal of Cleaner Production, vol. 137, no. 20, pp. 843-849, 2016.

[9] G. E. Vîlcu, "A geometric perspective on the generalized cobbdouglas production functions," Applied Mathematics Letters, vol. 24, no. 5, pp. 777-783, 2011.

[10] J. H. Cha and M. Finkelstein, "Justifying the Gompertz curve of mortality via the generalized Polya process of shocks," Theoretical Population Biology, vol. 109, pp. 54-62, 2016.

[11] O. Burger and T. I. Missov, "Evolutionary theory of ageing and the problem of correlated Gompertz parameters," Journal of Theoretical Biology, vol. 408, pp. 34-41, 2016.

[12] M. Liu, Theory and Method of Accident Risk Analysis, Peking University Press, 2011.
[13] L. Yun, F. Yun-xiao, and M. Xiao-chun, Risk Analysis and Safety Evaluation, Beijing, China, Chemical Industry Press, 2004.

[14] Z. Jing-lin and C. Guo-zhang, Safety System Engineering, Coal Industry Press, Beijing, China, 2001.

[15] P. Hong-jun, L. Xin-chun, and L. Yuan, “The non-linear optimal model of safety investment," Coal Engineering, vol. 12, pp. 70-72, 2016.

[16] T. Yi-lin and Y. Qing, "Urban disaster response capacity evaluation index system model design based on AHP-DELPHI method," Journal of Wuhan University of Technology (Transportation Science and Engineering), vol. 32, no. 1, pp. 168-171, 2008.

[17] Z. Zangenehmadar and O. Moselhi, "Prioritizing deterioration factors of water pipelines using Delphi method," Measurement, vol. 90, pp. 491-499, 2016.

[18] Z. Hou and P. Zhao, "Based on Fuzzy Bayesian Network of Oil Wharf Handling Risk Assessment," Mathematical Problems in Engineering, vol. 2016, pp. 1-10, 2016. 


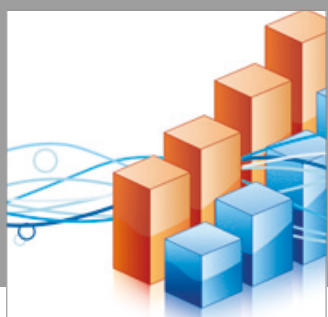

Advances in

Operations Research

vatersals

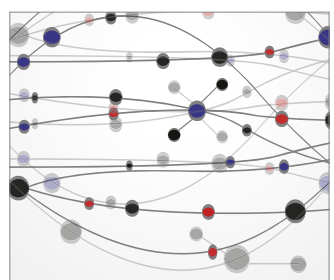

\section{The Scientific} World Journal
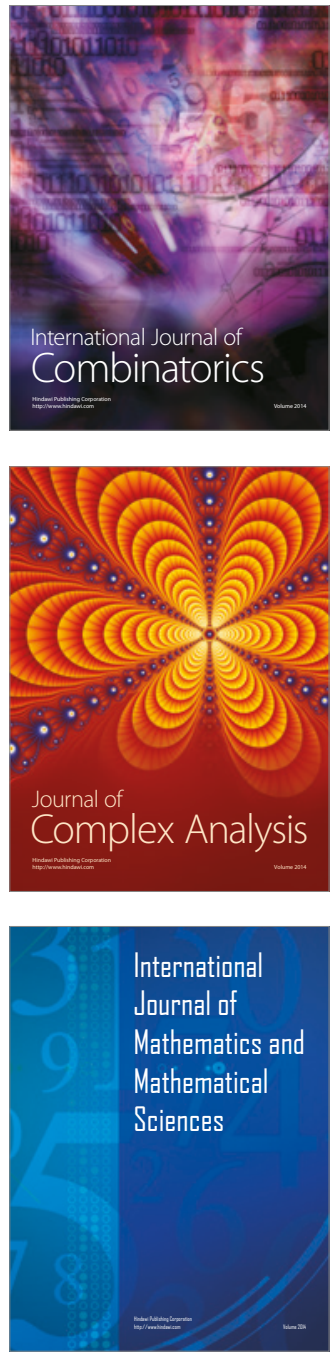
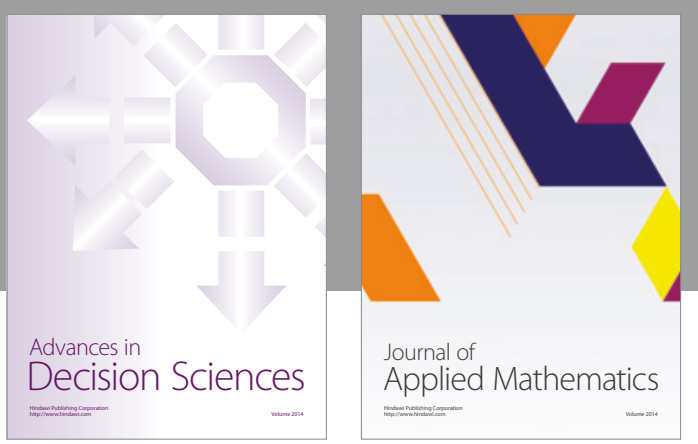

Algebra

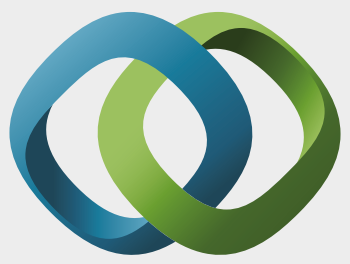

\section{Hindawi}

Submit your manuscripts at

https://www.hindawi.com
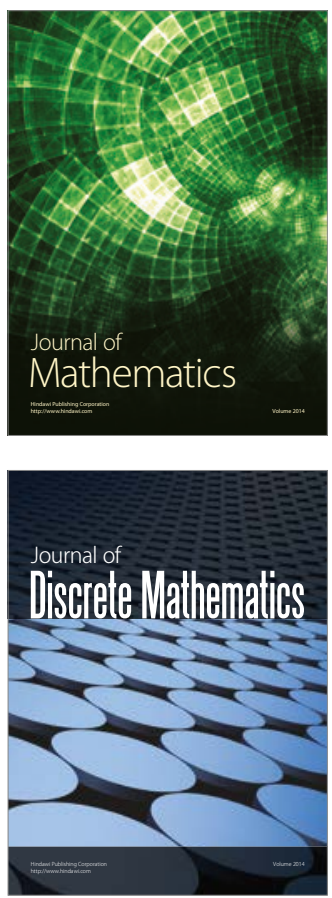

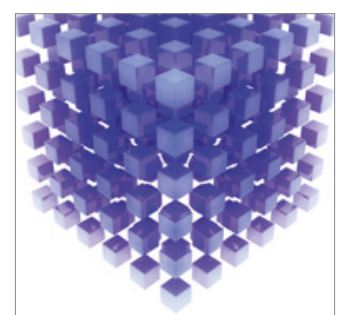

Mathematical Problems in Engineering
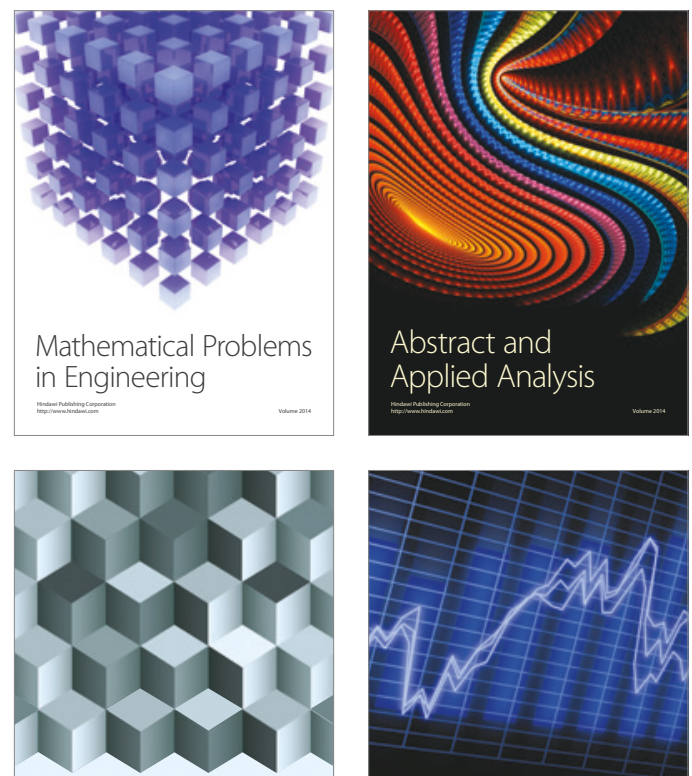

Journal of

Function Spaces

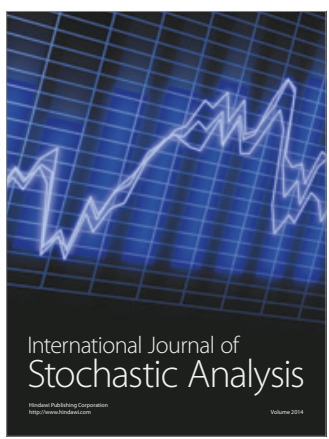

Probability and Statistics
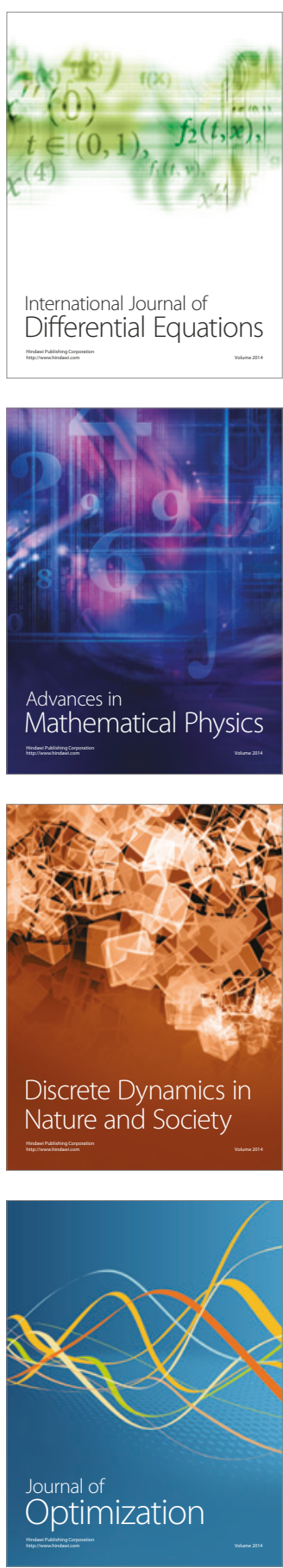